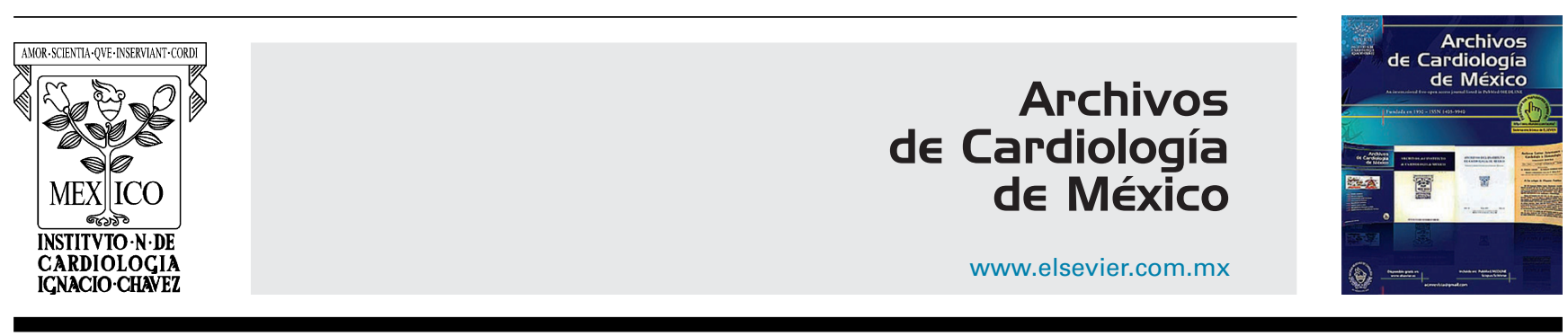

CARTA AL EDITOR

\section{Trastorno de conducción postoperatorio en el contexto de trombosis súbita de arteria coronaria derecha}

\section{Postoperative conduction disorder in the context of a sudden right coronary thrombosis}

\section{Sr. Editor:}

La estenosis valvular aórtica degenerativa es la valvulopatía más común en los países desarrollados, y el reemplazo valvular aórtico (RVA) su tratamiento convencional. La trombosis postoperatoria de las arterias coronarias es una complicación excepcional pero grave, que suele conducir hacia un infarto de miocardio masivo. Sin embargo, la trombosis de arteria coronaria derecha (ACD) también puede manifestarse en forma de un trastorno súbito de la conducción, lejos de los síntomas coronarios típicos. Esta presentación en el contexto de una cirugía reciente puede dar lugar a confusión en el diagnóstico y a un retraso en el tratamiento con consecuencias fatales.

Presentamos el caso de una mujer de 83 años de edad, hipertensa, sin otros factores de riesgo cardiovascular ni otros antecedentes relevantes, que es diagnosticada de estenosis valvular aórtica grave. Las arterias coronarias eran normales, y la paciente se encontraba en ritmo sinusal, sin alteraciones de la conducción asociadas, mostrando signos de hipertrofia ventricular izquierda con $\mathrm{T}$ negativas en derivaciones laterales (fig. 1A). La paciente fue sometida a un RVA por una prótesis de pericardio bovina Carpentier-Edwards Perimount Magna Ease n. ${ }^{\circ} 21$ (Edwards Lifesciences, Irvine, CA). No hubo complicaciones durante la cirugía ni en el periodo postoperatorio. Se inició tratamiento anticoagulante con acenocumarol en el segundo día de postoperatorio, si bien se detectaron niveles inadecuadamente bajos (INR $<2$ ) durante la primera semana postoperatoria. La paciente se mantuvo en ritmo sinusal estable durante todo el postoperatorio hasta el momento del alta. La paciente fue dada de alta sin complicaciones al octavo día tras la cirugía, con un adecuado nivel de anticoagulación (INR 2.6). El electrocardiograma al alta (fig. 1B) mostraba ritmo sinusal, sin mostrar alteraciones de la repolarización con respecto al ECG preoperatorio. El ecocardiograma de control postoperatorio previo al alta mostró una función biventricular normal y una prótesis aórtica normofuncionante (velocidad máxima $2.2 \mathrm{~m} / \mathrm{seg}$ y gradiente medio $14 \mathrm{~mm} \mathrm{Hg}$ ), además de descartar la presencia de derrame pericárdico o trombos intracavitarios.

En el momento de llegar a casa, y de forma súbita, la paciente sufrió un episodio brusco de mareo, sudoración y vómitos. Fue trasladada al Servicio de Urgencias; presentaba bradicardia, hipotensión y mostraba signos de bajo gasto cardíaco. No refirió dolor torácico o angina en ningún momento. La analítica convencional no mostró anomalías, salvo anemia típica postoperatoria, y la radiografía de tórax fue normal. Se realizó un electrocardiograma que reveló bradicardia, bloqueo de rama derecha y ritmo de la unión, así como una aparente elevación del ST de V1-V3 (fig. 2). La sintomatología se relacionó inicialmente con la presencia de un bloqueo cardiaco en el contexto de cirugía cardiaca reciente. Se realizó un ecocardiograma que descartó el taponamiento cardíaco, mostró una bioprótesis normofuncionante, pero objetivó una disfunción grave del ventrículo derecho, con función ventricular izquierda conservada. Se solicitaron niveles de enzimas cardiacas resultando una troponina $\mathrm{T}$ ultrasensible de $1,799 \mathrm{pg} / \mathrm{ml}$, una CK-Mb masa de $114.4 \mathrm{ng} / \mathrm{ml}$ y creatina-cinasa $669 \mathrm{U} / \mathrm{l}$. La elevación enzimática y evidente disfunción ventricular derecha hicieron sospechar una trombosis súbita de ACD, solicitándose una coronariografía urgente. En contraste con la coronariografía preoperatoria, que era normal y no presentaba anomalías coronarias (fig. $3 \mathrm{~A}$ ), se evidenció una trombosis masiva de la ACD y su rama ventricular (fig. 3B). Se consideró realizar cirugía de revascularización coronaria urgente. Sin embargo, debido a la inestabilidad extrema de la paciente, que hacía imposible trasladarla al quirófano, se intentó una trombectomía coronaria percutánea emergente. Se logró extraer parcialmente el trombo de la ACD (fig. $3 C$ y D) y restablecer, aunque de forma retrasada, el flujo a través de la misma (TIMI 2). Sin embargo, inmediatamente después del procedimiento la paciente presentó un episodio de hipotensión refractaria, falleciendo en la misma sala de hemodinámica a causa de un infarto ventricular derecho evolucionado.

La estenosis aórtica degenerativa es la valvulopatía más común en los países desarrollados, y el RVA su tratamiento estándar ${ }^{1}$. Una trombosis coronaria aguda después de un RVA es una complicación rara, pero frecuentemente fatal ${ }^{2}$. Por lo general la trombosis ocurre de forma aguda, durante la cirugía o postoperatorio inmediato en relación con una rotura de placa arterial, una disección coronaria, una obstrucción por 


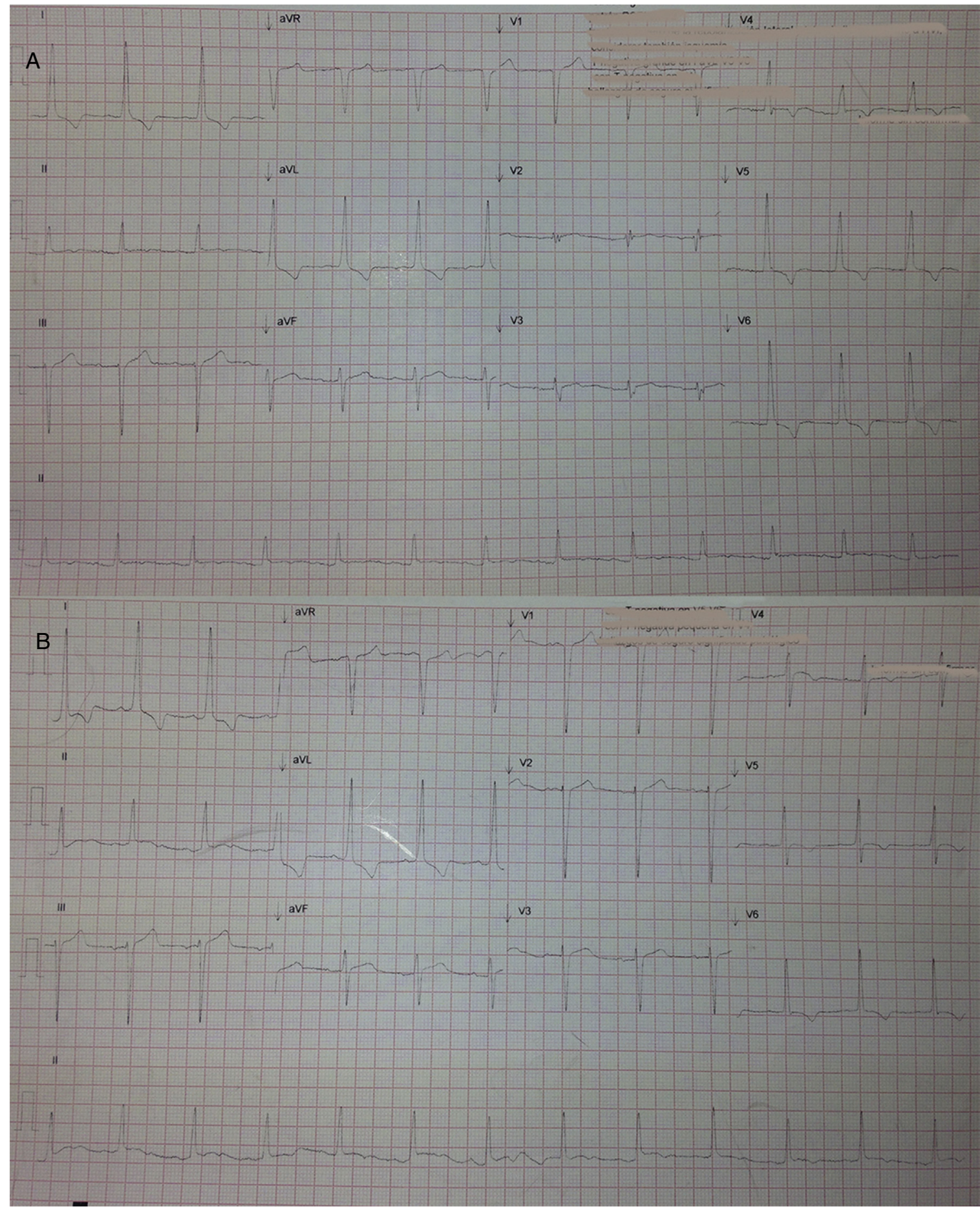

Figura 1 A. Electrocardiograma preoperatorio, mostrando ritmo sinusal con signos de hipertrofia ventricular izquierda. B. Electrocardiograma postoperatorio al alta hospitalaria.

la prótesis valvular implantada, una raíz aórtica de pequeño tamaño o en presencia de trastornos de coagulación, etc. La trombosis protésica resultado del daño endotelial tras la decalcificación valvular, la presencia de grietas o lesiones al nivel del anillo protésico puede ser origen de trombos que pueden migrar hacia las arterias coronarias ${ }^{3}$.

La mayoría de trombosis coronarias en el contexto de cirugía cardiaca se manifiestan intraoperatoriamente, de un forma aguda, como un infarto de miocardio y/o disfunción ventricular súbita, lo que facilita el diagnóstico y rápido tratamiento. Sin embargo, una trombosis coronaria subaguda a lo largo del período postoperatorio puede presentarse de una forma más abigarrada. Los trastornos del ritmo en el postoperatorio reciente de cirugía cardiaca no son infrecuentes. Sin embargo, ante un trastorno de la conducción tipo bloqueo de rama derecha de nueva aparición 


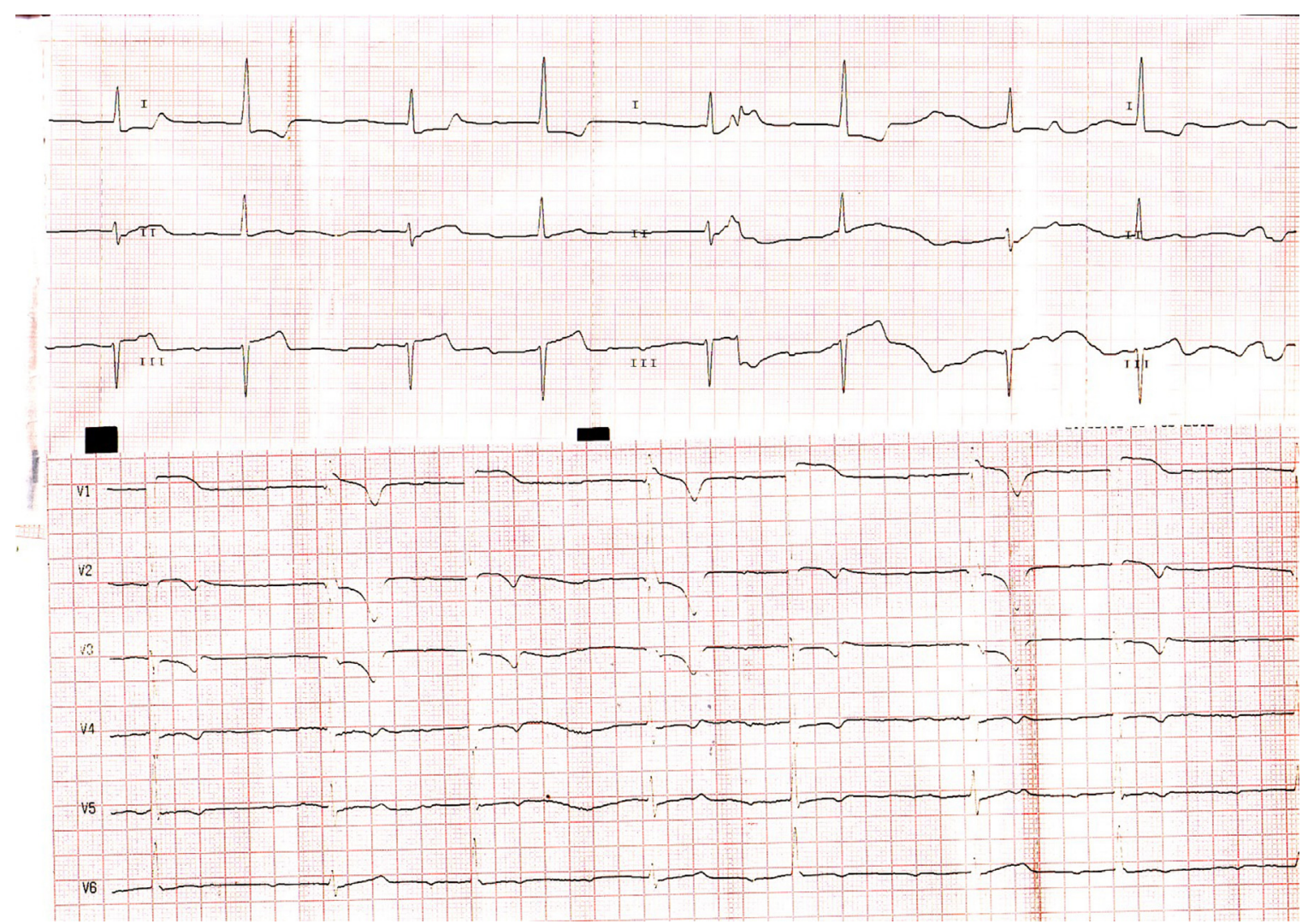

Figura 2 Electrocardiograma al ingreso mostrando bradicardia, bloqueo de rama derecha y ritmo de la unión, así como discreta elevación del segmento ST en derivaciones V1-V3.

debe existir un alto nivel de sospecha, ya que excepcionalmente puede ser reflejo de una oclusión de la arteria coronaria derecha. Esta forma de presentación poco común puede conducir a un retraso en el diagnóstico y a un tratamiento tardío con consecuencias fatales ${ }^{2,3}$. Además, en este contexto pueden estar presentes alteraciones de la repolarización tipo elevación del segmento ST en derivaciones precordiales V1-V3, que pueden ser reflejo de la oclusión de ramas ventriculares dependientes de la coronaria derecha ${ }^{4}$.

Por otra parte, la terapia de anticoagulación no es obligatoria después de un RVA por bioprótesis. Sin embargo, en nuestra institución está establecido un período de 3 meses de tratamiento anticoagulante postoperatorio con el objetivo de mantener anticoagulado al paciente el periodo en el que la presencia de arritmias perioperatorias es más frecuente y el paciente presenta una escasa movilización, siendo también los 3 meses el tiempo estimado hasta la completa endotelización de la prótesis y sutura de la misma.

Los niveles subterapéuticos de anticoagulación pueden relacionarse con la presencia de trombos intracardiacos como principal etiología de una oclusión o trombosis coronaria postoperatoria subaguda ${ }^{2-5}$. Sin embargo, en el caso que presentamos, esta no parece ser la causa de la trombosis súbita de arteria coronaria derecha, ya que la paciente no presentaba disfunción ventricular preoperatoria, ni aneurismas ventriculares, o enfermedades que pudieran predisponer a la formación de un trombo intracardiaco.
En el caso que describimos especulamos con la posibilidad de la formación de un trombo en torno a la prótesis aórtica, como causa de la posterior trombosis aguda de ACD. Por una parte, la formación del trombo pudo estar favorecida por diversos factores como la presencia de una raíz aórtica de pequeño tamaño, la presencia de la prótesis y sus suturas, la proximidad de los ostia coronarios a la misma, la más que segura incompleta endotelización del anillo protésico tras haber pasado escasamente 8 días de la cirugía, así como la confirmada existencia de niveles bajos e inadecuados de anticoagulación durante la primera semana postoperatoria en el contexto de un postoperatorio reciente, con periodos de bajo gasto cardiaco. Todos estos factores pudieron predisponer a la estasis sanguínea a dicho nivel y la formación del trombo en torno a la prótesis. Por otro lado, factores como la ausencia de calcificaciones en la raíz aórtica o lesiones coronarias significativas confirmadas por angiografía preoperatoria (fig. 2A), la ausencia de alteraciones de la conducción o repolarización al alta, la presencia de una prótesis aórtica normofuncionante en el ecocardiograma postoperatorio y en el reingreso, orientan a la migración súbita del trombo formado en la proximidad de la prótesis a través de la ACD debido a la mayor movilización de la paciente en el momento del alta.

Una vez establecido el diagnóstico de trombosis aguda de ACD, y en situación de estabilidad hemodinámica, el tratamiento médico (anticoagulación vs trombólisis) es una alternativa. Sin embargo, en situación de inestabilidad 


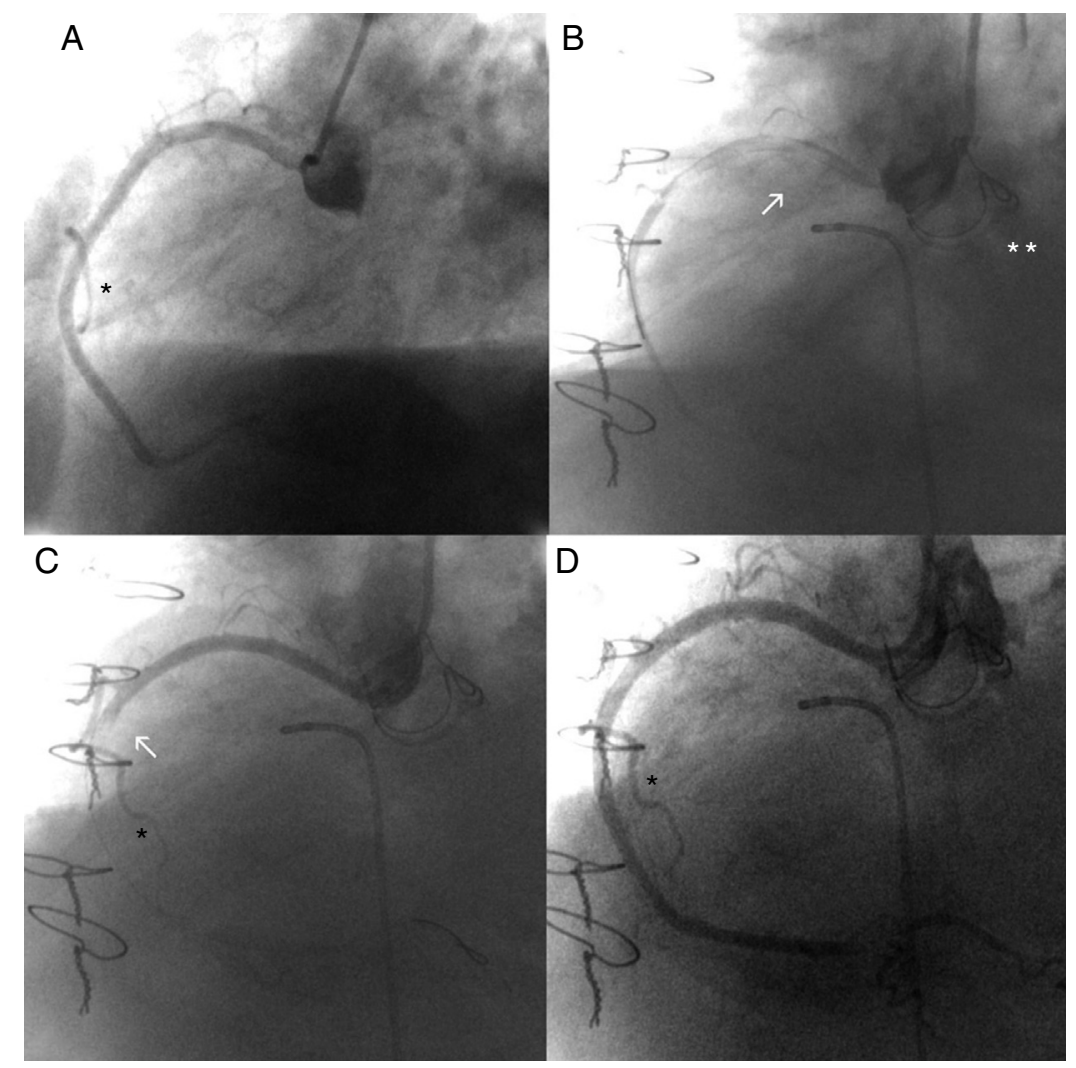

Figura 3 Coronariografía. A. Angiografía selectiva de arteria coronaria derecha preoperatoria mostrando la ausencia de lesiones en ACD y la presencia de pequeña rama ventricular dependiente de la misma (asterisco). B. Angiografía coronaria derecha postoperatoria mostrando la trombosis extensa de la ACD (flecha), así como la oclusión su rama ventricular. Se muestran los alambres de esternotomía y la prótesis valvular aórtica biológica (doble asterisco). C. Revascularización parcial de arteria coronaria derecha (flecha) y su rama ventricular (asterisco) tras trombectomía percutánea. D. Resultado final de la ACD (asterisco) tras la trombectomía percutánea.

hemodinámica y shock cardiogénico se precisa una intervención quirúrgica o percutánea emergente, con el fin de restablecer el flujo coronario de forma inmediata. Si bien, con o sin tratamiento, esta entidad presenta una alta tasa de mortalidad ${ }^{2,6-8}$.

\section{Bibliografía}

1. Bonow RO, Greenland P. Population-wide trends in aortic stenosis incidence and outcomes. Circulation. 2015;131:969-71.

2. Patel M, Bhangoo M, Prasad A. Successful percutaneous treatment of suspected embolic left main thrombosis in a patient with mechanical aortic valve. J Invasive Cardiol. 2011;23:e263-6.

3. Patanè F, Sansone F, Campanella A, et al. Acute bioprosthetic thrombosis immediately after aortic valve replacement. J Cardiovasc Med (Hagerstown). 2009;10:167-9.

4. Malekpour F, Castillo R. Surviving a rare event. Left main coronary artery occlusion. Tex Heart Inst J. 2012;39:594-5.

5. Marinakis A, Lampropoulos K, Precordial ST. Elevation due to isolated ventricular branch occlusion after stent implantation in the right coronary artery. Rev Esp Cardiol. 2009;62:1338-40.

6. Klein AJ, Casserly IP, Messenger JC. Acute left main coronary arterial thrombosis - a case series. J Invasive Cardiol. 2008;20:E243-6.

7. Hassink RJ, Haerkens-Arends HE, Daniels MCG. Extensive right coronary artery thrombosis. Neth Heart J. 2009;17:115-6.
8. Piana RN, Paik GY, Moscucci M, et al. Incidence and treatment of "no-reflow" after percutaneous coronary intervention. Circulation. 1994;89:2514-8.

María Elena Arnáiz-García ${ }^{a, *}$, Ana María Arnáiz-García ${ }^{\mathrm{b}}$, José María González-Santos ${ }^{a}$, María E. Bueno-Codoñer ${ }^{a}$, María Teresa González-Sánchez ${ }^{c}$, Javier López-Rodríguez ${ }^{a}$ y Javier Arnáiz ${ }^{\mathrm{d}}$

a Departamento de Cirugía Cardiaca, Hospital Universitario de Salamanca, Salamanca, España

b Departamento de Medicina Interna, Hospital

Universitario Marqués de Valdecilla, Santander, España

c Servicio de Cardiología, Hospital Universitario de Salamanca, Salamanca, España

d Servicio de Radiodiagnóstico, Aspetar-Orthopaedic and Sports Medicine Hospital, Doha, Qatar

* Autor para correspondencia. María Elena Arnáiz-García, Servicio de Cirugía Cardiaca, Hospital Universitario de Salamanca, Paseo de San Vicente 58-182, 37007, Salamanca, España. Tel.: +0034923291263; fax: +0034923291263.

Correo electrónico: elearnaiz@hotmail.com

(M.E. Arnáiz-García). 